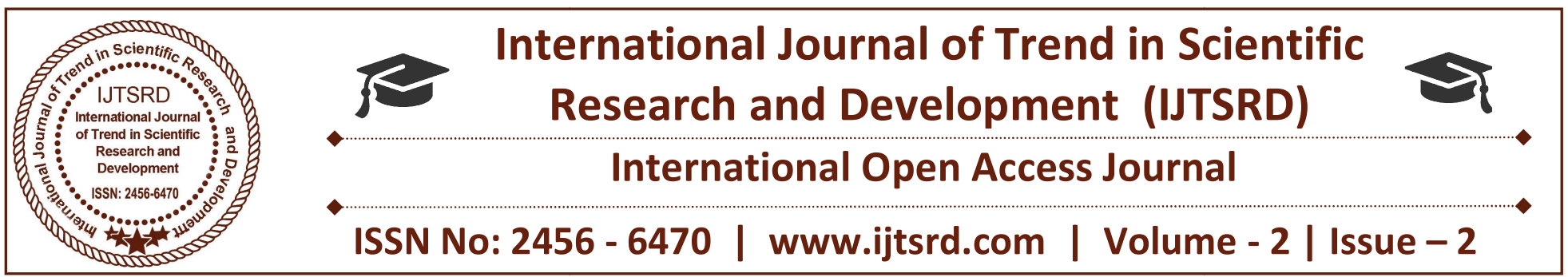

\title{
Providing Traditional Computational Facility through TV SetTop Box (PROTRACTS)
}

\author{
Mr. Prakruth H S \\ Assistant Professor, GSSS Institute of Engineering \\ \& Technology for Women, Mysore, India
}

\section{ABSTRACT}

The objective of the proposed Research work is to take the computer to everyone's home with DTH TV SetTop Box and TV set. The traditional dish cable TV is now replaced with Direct To Home (DTH) service using SetTop boxes. These SetTop boxes are embedded systems that process the signals from the DTH service to provide the broadcasting of the TV channels. SetTop boxes have processing power and considerable amount of computing capabilities. At present by using these set top boxes one can access only the service applications that are provided by the DTH like Video games, Video on demand, YouTube, Facebook, and Twitter.These computing capabilities can be used to perform low level computing applications like web browsing, File storage, Applications Downloading and installations Applications etc., which will be quite useful for all kinds of users.

Keywords: DTH, ERTOS, Kernel, SetTop Box we can use that computing power to perform some low level applications like browsing web, making documents storage etc., it will be quite useful for all kinds of users. So that no needs to buy a separate computer and all the computer accessories. So in this paper we are trying to make SetTop boxes are used to perform applications like File storage, programming computations by embedding RTOS and low level applications into the SetTop boxes. However, these computing facilities lay unutilized because necessary Embedded RTOS are not prebuilt into the SetTop Box. This paper aims to fill this gap by designing and incorporating an Embedded RTOS into the DTH SetTop Boxes to enable the user to have increased Ease of access, Universal UI, Huge number of apps and many other features. We are designed and developed an Embedded RTOS, which can be installable in DTH TV SetTop boxes. Once installed, these DTH TV Set Top boxes can be used to view TV programs as well as performing low level computational facilities as mentioned earlier.

\section{INTRODUCTION}

HE traditional dish cable TV is now replaced with Direct To Home (DTH) service using set top boxes. These SetTop boxes are embedded systems that process the signals from the DTH service to provide the broadcasting of the TV channels. SetTop boxes have processing power and considerable amount of computing capabilities. At present by using these set top boxes we can access only the service applications that are provided by the DTH like Video games, Video on demand, YouTube, Facebook and Twitter. If

\section{CUSTOMIZATION OF OS}

\section{Linux:}

Linux was originally developed as a free operating system for Intel x86-based personal computers. It has since been ported to more computer hardware platforms than any other operating system.

Linux also runs on embedded systems, devices where the operating system is typically built into the firmware and highly tailored to the system such as 
mobile phones, tablet computers, network routers, televisions and video game consoles; the Android system in wide use on mobile devices is built on the Linux kernel.

A distribution oriented toward desktop use will typically include the $\mathrm{X}$ Window System and an accompanying desktop environment such as GNOME or KDE Plasma. Some such distributions may include a less resource intensive desktop such as LXDE or Xfce for use on older or less powerful computers. So we can edit and remove unnecessary files and applications present in OS, so as to reduce the size and increase the performance of the system. As it is free, open source and can be run in different platforms and different devices as well so we have best choice of installing Linux in STB with a desktop environment of choice.

\section{Kernel:}

In computing, the kernel is the main component of most computer operating systems; it is a bridge between applications and the actual data processing done at the hardware level. The kernel's responsibilities include managing thesystem's resources, the communication between hardware and software components. Usually, as a basic component of an operating system, a kernel can provide the lowest-level abstraction layer for the resources, especially processors and $\mathrm{I} / \mathrm{O}$ devices that application software must control to perform its function. It typically makes these facilities available to application processes through inter-process communication mechanisms and system calls.

The main advantage of Linux kernel is, it is not originally designed to be portable. Linux is now one of the most widely ported operating system kernels, running on a diverse range of systems from the ARM architecture to the IBM Z/Architecture.

STB contains ARM architecture it is perfect and the best option to embed Linux.

\section{RELATEDWORK}

\section{Kernel Editing:}

The kernel is also free and open source. For customizing OS we should edit the kernel. It can be edited through the following process:
1. Download the latest version of the Linuxkernel from http://www.kernel.org.

2. Open the terminal and extract the kernel.

3. Configure the kernel. (make old config, menuconfig and qconfig/xconfig/gconfig )

4. Once configured, compile and install the kernel. (make \&\& make modules_install\&\&make install)

5. So the kernel is installed, now we need to make it bootable (mkinitrd -o initrd.img$<$ kernelversion $><$ kernelversion $>"$ )

6. Point the boot loader at the new kernel.

\section{IMPLEMENTATION}

\section{Deploying Ubuntu Core:}

Procedure:

1. Format target media: at least one partition should be ext 2 , ext 3 , or ext4

2. Unpack Ubuntu Core to ext $\{2,3,4\}$ partition

3. Install boot-loader

4. Install Linux

5. Add a local user and password for the user, then add the user to the adm and sudo groups.

6. If the Linux kernel requires modules, add these to $/$ lib/modules/ $($ uname -r) in the $\operatorname{ext}\{2,3,4\}$ file system .

7. Boot the target device.

8. Install any additional required software using aptget.

\section{Script to partition/format SDCards}

*\$ sudo ./omap3-mkcard.sh $/ \mathrm{dev} / \mathrm{sd}<\mathrm{x}>$

* \#!/bin/bash

if $[! " \$ 1 "=" / \mathrm{dev} / \mathrm{sda} "]$; then

unset LANG

DRIVE $=\$ 1$

if [ $b$ "\$DRIVE" ] ; then

dd if $=/$ dev/zero of $=\$ D R I V E$ bs $=1024$

count $=1024$

awk ' $\{$ print $\$ 5\}$ '

SIZE=`fdisk -1 \$DRIVE | grep Disk |

echo DISK SIZE - \$SIZE bytes

CYLINDERS='echo

\$SIZE/255/63/512 | bc

echo CYLINDERS - \$CYLINDERS

\{

echo $, 9,0 \mathrm{x} 0 \mathrm{C}, *$

echo,,,-

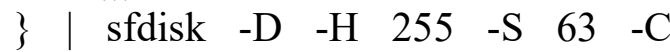

\$CYLINDERS \$DRIVE

mkfs.vfat -F 32 -n "boot" \$ $\{$ DRIVE $\} 1$

mke2fs -j -L "rootfs" \$ $\{$ DRIVE 2 
fi

Step by Step Instructions to format SD card Since putting a Linux file system on a FAT32 partition is problematic, it is recommended to also create a 2nd partition.

- Insert your SD card into your Linux box

- Do not mount it

The card shows up as $/ \mathrm{dev} / \mathrm{sd}^{*}$. To identify the card, you can either do:

dmesg | grepsd[a-z]. You will see something like [172407.246308] sdb: sdb1 sdb2 so drive is /dev/sdb

- $1 \mathrm{~s} / \mathrm{dev} / \mathrm{sd}^{*}$ before and after plugging SD card and find created devices

Delete existing Partitions:

\# Command (m for help): $\mathrm{d}$

Partition number (1-4): 1

Command ( $\mathrm{m}$ for help): $\mathrm{d}$

Partition number (1-4): 2

Configure SD Card:

Command ( $\mathrm{m}$ for help): $\mathrm{x}$

Expert command ( $\mathrm{m}$ for help): $\mathrm{h}$

Number of heads (1-256, default 30): 255

Expert command ( $\mathrm{m}$ for help): $\mathrm{s}$

Number of sectors (1-63, default 29): 63

Warning: setting sector offset for DOS compatibility

Expert command ( $\mathrm{m}$ for help): c

Number of cylinders (1-1048576, default 2286):

$<$ new_cylinders calculated from above $>$

Configure SD Partitions:

1. Expert command ( $m$ for help): $r$

2. Command ( $\mathrm{m}$ for help): $\mathrm{n}$

3. Command action

4. e extended

5. p primary partition (1-4)p

6. Partition number (1-4): 1

7. First cylinder (1-123, default 1):

8. Using default value 1

9. Last cylinder or + size or + sizeM or + sizeK (1123, default 123): $+64 \mathrm{M}$

10. Command ( $m$ for help): $n$

11. Command action

12. e extended

13. $\mathrm{p}$ primary partition $(1-4) \mathrm{p}$

14. Partition number (1-4): 2

15. First cylinder (10-123, default 10):

16. Using default value 10
17. Last cylinder or + size or + sizeM or + sizeK (10123, default 123):

18. Using default value 123

Check Partition Table.

* Command ( $\mathrm{m}$ for help):

Disk /dev/sdc: 993 MB, 993001472 bytes

255 heads, 63 sectors/track, 120 cylinders

Units $=$ cylinders of $16065 * 512=8225280$ bytes

Disk identifier: 0x00000000

Device Boot Start End Blocks Id System:

$/ \mathrm{dev} / \mathrm{sdc} 1 * 1972261 \mathrm{c}$ W95 FAT32 (LBA)

/dev/sdc2 $10120891607+83$ Linux

Command ( $\mathrm{m}$ for help): w

The partition table has been altered!

Calling ioctl() to re-read partition table.

Creating a mount point:

+ mkdir $/ \mathrm{tmp} / \mathrm{mmcl}$

\# mkdir $/ \mathrm{tmp} / \mathrm{mmc} 2$

sudo mount $/ \mathrm{dev} / \mathrm{sdc} 1 / \mathrm{tmp} / \mathrm{mmc} 1$

sudo mount $/ \mathrm{dev} / \mathrm{sdc} 2 / \mathrm{tmp} / \mathrm{mmc} 2$.

\section{ODROID-X:}

ODROID-X is a micro sized open development platform which is powered by Exynos4412. 1.4Ghz ARM Cortex-A9 Quad Cores show PC-like performance with compatible architecture of android mobiles, STB's .

Configuration:

* ARM processor Cortex-A9 $1.4 \mathrm{GHz}$

Mali-400 MP Core

* 6 x High Speed USB2.0 Host Port

10/100Mbps Ethernet with RJ-45 LAN Jack

\section{USES:}

The OMAP boards have better configuration but with low compatibility with TV.

Beagle board is compatible with TV but has low configuration.

ODROID-X is compatible and has good configuration. So, now the best choice among the boards is ODROID-X board.

\section{CONCLUSION}

we can use the computing power of STB to perform some low level applications like browsing web, 
making documents storage etc., it will be quite useful for all kinds of users. So that no needs to buy a separate computer. So in this research work we are trying to make set top boxes are used to perform applications like File storage, programming computations by embedding RTOS and low level applications into the set top boxes.

\section{REFERENCES}

1. Lalit Kumar, Rajesh Kushwaha, Rishi Prakash: "Design \& Development of small Linux Operating System for Brower Based Digital set Top Box", 2009 First International Conference on Computational Intelligence, Communication Systems and Networks, pp. 277-281

2. Implementation of internet browsing on Set Top Box using ARM-LinuxNirmala, L; Manvi, Sunil Kumar S.; Reddy, R.Sreenivasa IET CONFERENCE PUBLICATIONS 